\title{
Optimal lipid metabolism with disease-preventing functional foods
}

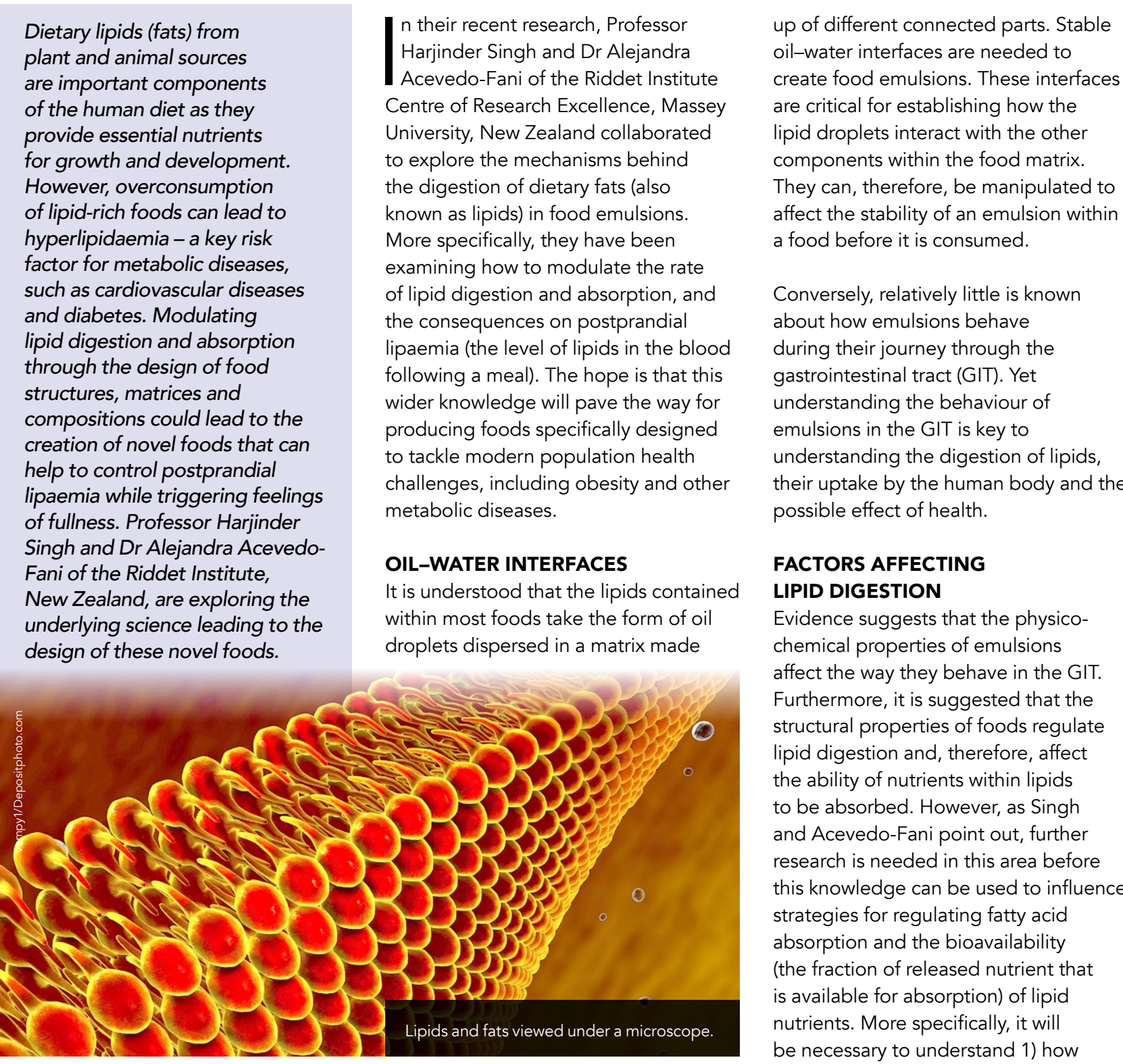

Dietary lipids (fats) from plant and animal sources
are important components of the human diet as they provide essential nutrieyts for growth and development However, overconsumption of lipid-rich foods can lead to hyperlipidaemia - a key risk factor for metabolic diseases, such as cardiovascular diseases and diabetes. Modulating lipid digestion and absorption structures design of food compositions could lead to the creation of novel foods that can help to control postprandial lipaemia while triggering feelings of fullness. Professor Harjinder Singh and Dr Alejandra AcevedoFani of the Riddet Institute, New Zealand, are exploring the underlying science leading to
design of these novel foods.

n their recent research, Professor Harjinder Singh and Dr Alejandra Centre of Research Excellence, Massey University, New Zealand collaborated to explore the mechanisms behind the digestion of dietary fats (also known as lipids) in food emulsions. More specifically, they have been examining how to modulate the rate of lipid digestion and absorption, and the consequences on postprandial lipaemia (the level of lipids in the blood following a meal). The hope is that this wider knowledge will pave the way for producing foods specifically designed challenges, including obesity and other ches

OIL-WATER INTERFACES It is understood that the lipids contain droplets dispersed in a matrix made

LIPID DIGESTION

GESTION

Evidence suggests that the physicoaffect the way they behave in the GIT. Furthermore, it is suggested that the structural properties of foods regulate lipid digestion and, therefore, affect the ability of nutrients within lipids to be absorbed. However, as Singh and Acevedo-Fani point out, further research is needed in this area before this knowledge can be used to influence strategies for regulating fatty acid absorption and the bioavailability the fraction of released nutrient that is available for absorption) of lipid nutrients. More specifically, it will
be necessary to understand 1) how

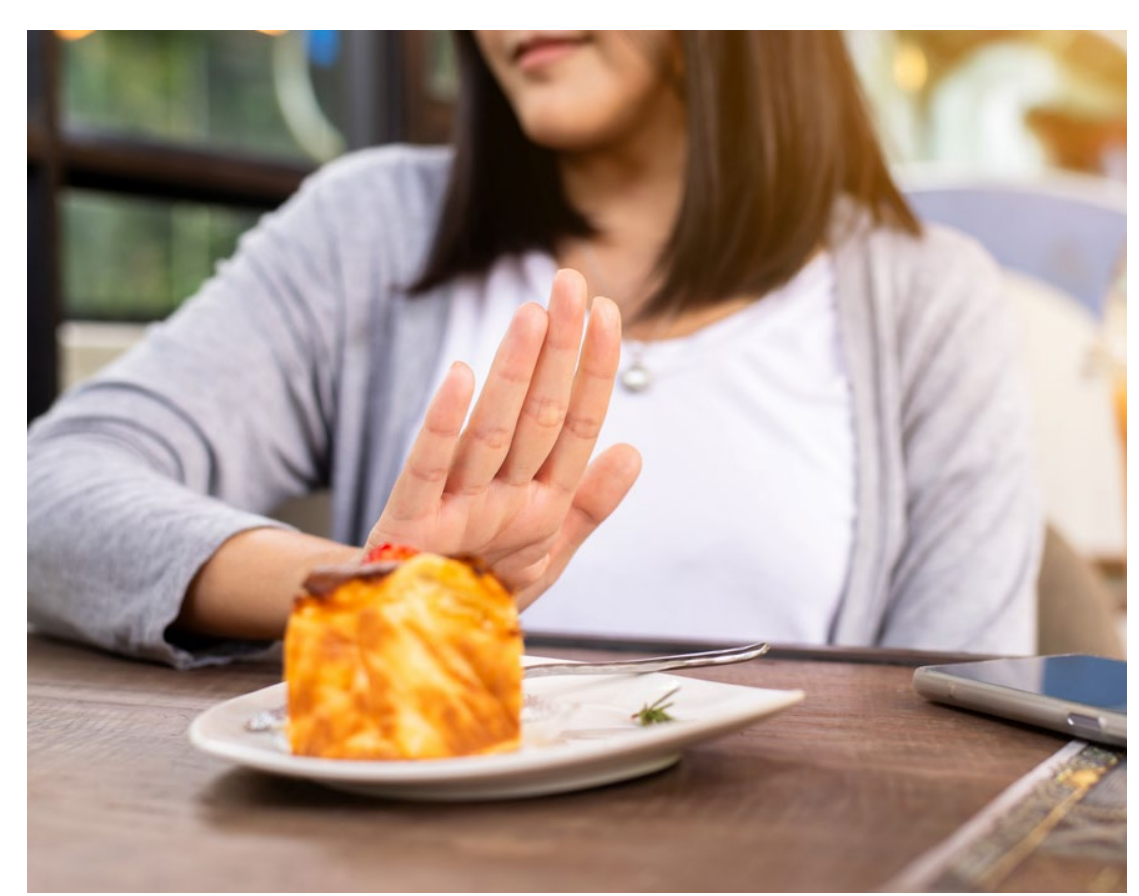

The research could lead to the formulation of novel foods which control postprandial lipaemia while the makeup of interfaces of the lipid towards the gut epithelium, where they the body uses to break down dietary lipids); 2) how bile salts (liver secretions that aid to break down lipids) dissupt the original oil-water interfaces; and 3) what the state of the droplets is just before absorption.

MEMBRANE TRANSPORT OF LIPIDS Emulsion interface functions as a protective barier that controls the rate at which lipids are digested. It is also possible to select certain elements of emulsions that can bind to digestive ability to solbilise and trence the products of lipid digestion.

Additionally, the intestinal lumen contents can be made thicker and stickier by adding gelling or thickening agents in the emulsions. This slows the transport of enzymes towards the emulsion droplet and the diffusion (moving from an area of higher concentration to an area of lower

FATS AND FUNCTIONAL FOODS Functional foods with reduced patty acid bioavailability could be hyperlipidaemia, who are at high risk of cardiovascular disease and obesity. Indeed, there is awareness of the need, among both consumers and the food industry, to address the serious public health issue of the obesity epidemic. will be in tackling this problem, wutrition in nutritional value of dietary fat, which source of essential fatty acids.

FACTORS AFFECTING FULLNESS MRI scans have been used to explore the relationship between how emulsions on feelings of fullness. This research. has improved understanding of how the contents of the GIT are distributed as well as stomach emptying. Oil-in-

The structural properties of foods regulate lipid digestion and affect the ability of nutrients within lipids to be absorbed. cidity of the stomach appear to slow the rate of emptying while increasing hormone which signals fullness) after eating. Conversely emulsions that bre down in these acid conditions result in rapid layering of fat in the stomach, which speeds up emptying and releases less CCK.

TAILORING EMULSIONS FOR LIPID METABOLISM

As our understanding of how different food emulsion systems interact with a range of biochemical and biophysical environments in the GIT has been increasing over the last decade, this has created the potential to design and sions with specific structures and properties. These emulsions can digestion, and absorption. However, most existing studies in this area have relied upon comparatively simple

model systems and lab-based digestion

models. Furthermore, attempts to alter the interfacial layers of emulsions to affect lipid digestion have had limited success because of the high levels of surface activity of bile salts. Additionally, while encapsulating emulsion droplets inside hydrogel particles has been shown to delay lipid digestion, this method is unlikely to be widely applicable in real foods.

MANIPULATING OIL DROPLETS Manipulating the physical state and

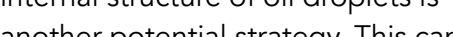
be achieved by altering the ratio of solids to liquids (lipids). However this could lead to part of the lipid (solid, crystalline fat that melts above body temperature) being completely indigestible. Therefore, as Singh and Acevedo-Fani highlight, before approving this strategy for use in foods, it is necessary to understand any potential physiological effects of allowing these undigested lipids to enter the large intestine. What is more, in terms of human health, it would not be advisable to increase the saturated and trans fat content of foods.

DESIGNER FOOD MATRICES Controlling the rate at which the stomach empties lipids is the most 
digestion. This is primarily affected by the interaction between droplets and food matices. ll is possible to design to the stomach varying interactions with oil droplets to affect lipid digestion. However more research is needed to determine the behaviour of different food materials and natural foods within the stomach. Singh and Acevedo-Fani recommen using more advanced methods that can accurately monitor the emptying of the stomach contents.

INTERCONNECTED

FOOD COMPONENTS

Most foods are complex in structure and composition. The emulsion is just one of the components of a food. It can help to form the structures of more and polysaccharides, which then form ma polysacchandes, which then form a droplets, such as in yoghurt, processed cheese, and other gels. However, the way in which emulsion structures designed to modulate lipid digestion behave in these complex systems is not yet fully understood. Therefore, when designing complex foods in future, it will be important to ensure that the emulsion system behaves in the desire way within the body.

\section{MANIPULATING}

MULTIPLE INTERACTIONS

Another approach could involve

manipulating the interactions betwe

a range of components within the

complex food syster in during digestion. As understanding of the complexity of lipid digestion

relation to food systems increases, it

will eventually be possible to design

functional foods with specific lipid digestion profiles. These foods will induce specific responses within the body, helping to increase feelings of fullness after eating and reduce the prevalence of obesity and cardiovascular disease risk. First, as Singh and Acevedo-Fani highlight, more human trials are needed to determine the long-term effects on health of delaying or controlling lipid digestion.

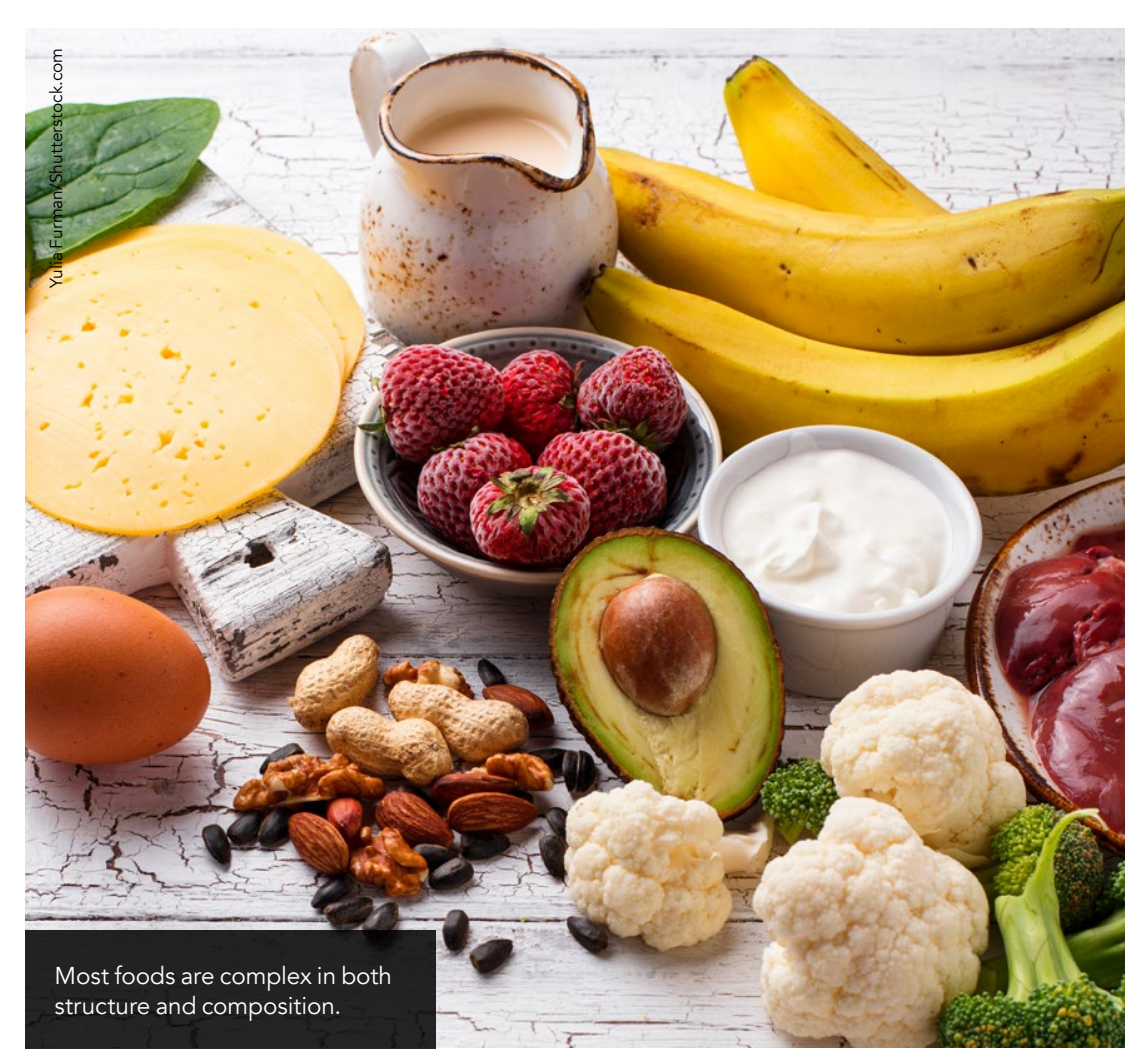

of biopolymers, such as proteins and polysaccharides, relating to the physiology of digestion in the stomach. Along with lipids, foods are made up of a wide range of biopolymers, and the ways in which these biopolymers interact during food processing an formulation play a part in creating physical and sensory properties of food biopolymers within the stomach and

Controlling the rate at which the stomach empties lipids is the most realistic strategy for delaying lipid digestion.

products. These structures have been used to develop new functions and textures in processed foods, along with matrices to encapsulate and deliver

flavours and nutrients. BREAKDOWN AND CREATION OF FOOD STRUCTURES

Through their research, Singh and Acevedo-Fani found that the way in which biopolymer interactions work during digestion, and how they impact on stomach emptying and nutrient absorption, is not yet fully understood. What is known, however, is that the disintegration of food stuc physical associating them with their functional performance

FURTHER RESEARCH As Singh and Acevedo-Fani explain, the ultimate aim of understanding lipid

the formation of entirely new structures. , allows macromolecules to interact. which can result in the formation of gels determining associated with hyperlipidaemia whout reducing the health benefits wing thes of the prots ant digestion will facilitate the production of foods designed for specific health characteristics. Moving forward, intensive clinical studies are functional foods.

\section{Behind the Research}

Professor

Harjinder Singh

Dr Alejandra Acevedo-Fani

E: h.singh@massey.ac.nz T: +646951 7317

Research Objectives

Professor Harijnder Singh and Dr Alejandra Acevedo-Fani research lipid digestion.

\section{Detail}

Harjinder Singh

Director

Riddet Institute (PN 445)

Massey University
Private Bag 11222

Private Bag 11222
Palmerston North

NewZealand

Bio

Harjinder Singh is Distinguished

Professor, and the Director of the

Riddet Institute (Centre of Research

food proteins, food colloids, and food structures and digestion interface. He has published over 350 research papers the Roy Socity of New Zealond and the Royal Society of New Zealand and Food Science and Technology and has received several internatogy, and has including the New Zealand Prime

Minister's Science Prize.

Dr Alejandra Acevedo-Fani is a Research Officer at the Riddet Institute, New Zealand. Her research involves
Excellence), Massey University, New

investigating modifications of the food structure in the digestive tract, particularly bioactive compounds-

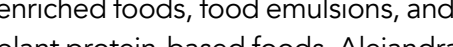
is also a Marie Curie Fellow at the International lberian Nanotechnology Laboratory in Portugal jointly with her role at the Riddet Institute.

\section{Funding}

Riddet Institute Centre of Research Excellence, Tertiary Education

\section{References}

Acevedo-Fani, A, Singh, H, (2021) Biophysical insights into modulating lipid digestion in food emulsions. Progress in Lipid Research, 85. dol.ogrto:

Acevedo-Fani, A, Singh, H, (2021) Biopolymer interactions during gastric digestion: Implications for nutrient delivery. Food Hydrocolloids, 116. doi.org/10.1016/2 foodhyd.2021.106644

Singh, H, Ye, A, Horne, D, (2008) Structuring food emulsions in the gastrointestinal tract to modify lipid digestion. Progress in Lipid Research, 48(2), 92-100. doi.org/10.1016/ plipres.2008.12.001

Viddet Institute

\section{Personal Response}

What action are food industry stakeholders taking in response to your research findings?

II The area of lipids and health has been of major interest for the global food industry for many decades. To date, the focus has been on developing food products with low fat content and in particular low levels been to match the consumers' organoleptic properties of fully fat products. Some high fat products with added plant sterols are commercially available to reduce the absorption of cholesterol. The concept of controlling the rate of lipid digestion through food structure design is relatively new and has not been yet translated into
commercial products, to our knowledge. of saturated and trans fats. However, the challenge has 